\title{
High time for a new journal with an Asian focus
}

\author{
Roger R. Stough ${ }^{1,2}$
}

(C) The Japan Section of the Regional Science Association International 2017

It was my great pleasure to learn about the new Asia-Pacific Journal of Regional Science (APJRS) that was recently initiated as a Springer publication. Given all the growth in high quality regional science scholars in Asia over the past generation it is high time that the field has this new journal with an Asian focus.

The dynamism of the Asian economies and all the new innovations that have and are occurring in how to drive an economy through the various stages of growth faster than the world has ever seen before promises a constant and growing flow of exceptional papers not only from Asian scholars but also from researchers from other parts of the world. Moreover, the development experiments that are occurring at the sub-national regional levels in Japan, China, Taiwan and Korea, for example, offer super targets for informative and provocative research that will find its way to the APJRS and thus help to inform the rest of the world about these innovations. In so doing it will propel the journal into a major leadership role in regional science.

Again, the startup of the APJRS is great news and I look forward to its rapid development and I promise to help in its evolution to a top journal in the field.

Roger R. Stough

rstough@gmu.edu

1 Schar School of Policy and Government, George Mason University, Arlington, VA, USA

2 Strategic Development, The Regional Science Academy, Stockholm, Sweden 